\title{
Ventaja competitiva: EI reto de las PyME en la industria del calzado
}

\author{
Ojeda Gómez, Julieta*
}

\section{Resumen}

Las reformas económicas del Reyno Unido y Mexico ocurridas en los años ochenta, la industria del calzado también experimentó a nivel internacional una considerable reestructuración. Esto redefinió la base competitiva de muchos países productores de calzado. El objetivo de este artículo es analizar el desarrollo de ventaja competitiva en las pequeñas y medianas empresas en la industria del calzado británica y mexicana. Mediante un enfoque histórico se estudiaron empresas creadas y/o que sobrevivieron el periodo 1979-1999. Se elaboraron diez estudios de caso cuyos datos fueron recopilados mediante entrevistas y clasificados según representaran interacciones y cambios ocurridos en el papel del empresario, en los recursos de las empresas o interacciones con las instituciones de apoyo. Los resultados indican que los recursos básicos de las empresas fueron los cimientos para el desarrollo de una ventaja competitiva mínima necesaria para su supervivencia. Superada esta etapa, el mejoramiento de su posición competitiva ocurrió por la adopción de estrategias complementarias. Esto sugiere que las empresas de menor tamaño pueden desarrollar ventaja competitiva de una manera particular a diferencia de las empresas de mayor tamaño.

Palabras clave: Industria del calzado, México, Reino Unido, ventaja competitiva.

\section{Competitive Advantage: the Challenge for Small and Medium Businesses in the Footwear Industry}

\section{Abstract}

Along with the economic reforms that took place in the United Kingdom and Mexico in the 1980 s, the footwear industry experienced considerable re-structuring on the international level, redefining the competitive basis for many footwear-producing countries. The objective of this article is to analyze the development of competitive advantage in small and medium businesses in the British and Mexican footwear industry. Using a historic approach, businesses that were created and/or survived

Recibido: 09-04-07. Aceptado: 26-07-07

La autora es investigadora de la Facultad de Contaduría y Administración de la Universidad Nacional Autónoma de México. Correo electrónico: jojeda@correo.fca.unam.mx 
the period from 1979 to 1999 were studied. Ten case studies were developed; data was compiled using interviews and classified according to the interactions and changes occurred in the roles of the businessman, company resources or interactions with support institutions. Results indicated that a company's basic resources were the foundation for developing the minimum competitive advantage needed for survival. Once this stage had been surpassed, improvement of competitive position occurred due to the adoption of complementary strategies. These results suggested that smaller companies can develop competitive advantage in a particular manner, different from larger companies.

Key words: IFootwear industry, Mexico, United Kingdom, competitive advantage.

\section{Introducción}

Esta investigación se enfoca en el estudio del desarrollo de la ventaja competitiva como reto importante para las pequeñas y medianas empresas (PyME), en la industria del calzado. A principios de los años ochenta, muchos países adoptaron reformas económicas que trajeron cambios drásticos en la forma en que operaban la economía nacional y las empresas. Algunas de estas reformas tuvieron por objetivo la apertura de los mercados e instituciones así como la eliminación del intervencionismo público. Estas condiciones existieron en México cuando surgió la liberalización de la economía en los ochenta dejando a las empresas con el reto de enfrentar a la competencia proveniente del exterior y de operar su negocio eficientemente. En el Reino Unido ocurrieron los procesos de desindustrialización y desregulación industrial en las décadas de los setenta y ochenta creando nuevas condiciones de negocios.

La reducción gradual del sector manufacturero y la eliminación del control gubernamental directo sobre diversas actividades pretendían, respectivamente, la transición hacia una economía de servicios y la operación más eficiente de los mercados. Aquellas empresas en industrias de uso intensivo de mano de obra se vieron en la necesidad de adaptarse a la nueva división internacional del trabajo en la que los países de bajos costos empezaron a jugar un papel importante.

En este mismo periodo, la industria del calzado tuvo una reestructuración significativa internacionalmente debido a que muchos países redefinieron su base competitiva para adaptarse a los cambios en la industria. El surgimiento de los países de mano de obra barata en el mercado internacional erosionó la ventaja comparativa de los productores en países industrializados por lo que la cadena global reestructuró (Andrews et al., 1998 y Vokurka et al., 2002).

Ante estos cambios, las empresas de todos tamaños en México y en el Reino Unido se vieron expuestas a la competencia internacional. Su reto era desarrollar ventajas competitivas que las mantuvieran en el mercado y adaptarse a las nuevas condiciones de negocios en la industria. Este reto tenía una doble implicación. La primera era asegurar la supervivencia de la empresa mientras ocurría la adaptación a las nuevas condiciones del entorno. La segunda era encontrar una posición competitiva que permitiera a la 
empresa competir exitosamente a nivel nacional e internacional. Ante esta condición, muchas PyME no sobrevivieron pero otras empezaron a tener una participación notable. El estudio de las PyME exitosas pudiera sugerir el desarrollo de una manera particular de lograr ventajas competitivas, cuya explicación puede diferir de los modelos tradicionales.

Estos últimos tienden a basarse en la experiencia y características de las empresas grandes por lo que no necesariamente se ajustan a las condiciones de las PyME. Éstas poseen limitaciones inherentes a su tamaño, tales como recursos limitados, nulo poder de mercado, difícil acceso a ciertos recursos y vulnerabilidad.

La literatura referente a la ventaja competitiva tiende a explicar este proceso mediante el análisis de diversos factores considerados individualmente y aislados de eventos particulares del ambiente. Estas omisiones han pasado por desapercibido el contenido y el proceso de adaptación estratégica. Además, otras investigaciones han hecho hincapié en la necesidad de estudios a nivel empresa y específicos a un país que aborden los vacíos existentes (Ray, 2003).

Estos "espacios" en la literatura motivaron el desarrollo de este proyecto cuyos objetivos principales son el análisis de la forma en que las PyME en México y El Reino Unido desarrollaron ventajas competitivas durante un periodo de cambios drásticos a nivel industria y en la economía de ambos países (1979-1999), así como identificar similitudes y diferencias.

Para efectos de este proyecto los términos "ambiente" y "entorno" son utilizados indistintamente y se refieren a aquellos eventos que cambian tendencias y crean oportunidades y amenazas para las empresas. El ambiente incluye aspectos a nivel macro y micro. A nivel macro encontramos cambios en el ámbito económico, político, tecnológico, social, cultural y demográfico. En el nivel micro están todos aquellos factores presentes en la operación diaria de la empresa, como son los proveedores, consumidores, distribuidores, competidores, la comunidad en la que se encuentran inmersas, instituciones gubernamentales, entre otras.

\section{Marco teórico}

La estrategia en las PyME puede representar un esfuerzo de adaptación a los cambios en el ambiente (Knight, 2000). La capacidad de adaptación de una empresa está determinada por su dinámica interna basada en el aprendizaje y acumulación de experiencia ganada con el tiempo (Alvarez y Busenitz, 2001). Las ventajas y dificultades de las PyME influyen en sus estrategias, mismas que no son formuladas necesariamente de manera consciente, o se encuentran escritas formalmente, o expresas en términos específicos (Quinn, 1996).

Así, las estrategias de las PyME pueden ser el resultado de un cálculo deliberado o de la confusión, por accidente, intuición o inercia (Whittington, 1993). La escuela de configuración, postulada por Mintzberg et al. (1998), explica acertadamente la elaboración de estrategias en las PyME. Esta escuela señala que la estrategia puede ser un plan, una posición, un patrón, una perspectiva o una pauta de acción, dependiendo de la situación y 
tiempo específicos en que ocurre con el fin de lograr estabilidad. Esta dinámica puede facilitar nuevas combinaciones de recursos y capacidades al amalgamar creativamente los recursos propios 0 a los que puede tener acceso la empresa (Herrera et al., 2002).

El concepto de ventaja comparativa basado en los costos de mano de obra no considera otros aspectos que han dado ventajas a las PyME. Para desarrollar ventaja competitiva, las PyME dependen principalmente de los medios disponibles a su alcance, ya sea que éstos se encuentren dentro de la misma empresa o disponibles a través de instituciones públicas o privadas. Muchas de sus ventajas han derivado de su flexibilidad, innovación, capacidad de adaptación, entre otras.

Estos factores tampoco han sido considerados en los modelos tradicionales que explican la ventaja competitiva, puesto que tienden a basarse en la experiencia y características de las empresas grandes. Estos modelos ponen especial énfasis en la habilidad que tienen estas empresas para influir en su entorno o ejercer su poder de mercado. Por esta razón, estos modelos no necesariamente se ajustan a las condiciones de las PyME. La postura del autor subraya la importancia de considerar y estudiar a las PyME bajo sus propias condiciones y para ello, se propone un enfoque alterno que explique la ventaja competitiva de las PyME.

Este enfoque considera que la ventaja competitiva resulta de un proceso dinámico debido a los cambios en, y la influencia ejercida por, el ambiente, el apoyo de diversas instituciones y los cambios que ocurren en el papel del empresario y/o los recursos de la empresa. Todos factores son dinámicos e interactúan entre si y también necesitan adaptarse a las demandas del entorno. Por tanto, este proceso requiere adaptación, aprendizaje, monitoreo del entorno, cooperación y el desarrollo de estrategias que ayuden a las PyME a lograr sus objetivos. Además, se considera que la adaptación es un medio de mantenerse competitivo (Man et al., 1998).

Cada PyME desarrolla su propio potencial basado en sus recursos estratégicos (valiosos), solos o en combinación, para adecuarse a las demandas del entorno (Collis y Montgomery, 1995; De Oliveira y Evaldo, 2003). Estos recursos representan aquellos activos tangibles e intangibles que neutralizan amenazas (Barney, 1991). Este enfoque se basa en la Teoría de Recursos misma que enfatiza la importancia de que el empresario esté consciente de las potenciales sinergias que se pueden crear entre los recursos y cómo utilizarlos adecuadamente en la formulación de estrategias (Barney, 1986).

Las actitudes del empresario influyen en la forma en que se explota la capacidad de la empresa para competir convirtiéndose en un activo esencial para impulsar las estrategias y "manejar" proactivamente el entorno (Knight, 2000). Esto requiere la habilidad del empresario para descubrir y crear valor agregado mediante la combinación de recursos que obtengan ventaja de las oportunidades (Kickul y Gundry, 2002). Las percepciones que se tengan del ambiente influirán en el comportamiento que el empresario adoptará tanto para responder al entorno como para diseñar estrategias efectivas. 
Adicionalmente, los enfoques cognitivos del empresario tendrán sus fortalezas y debilidades en determinados entornos competitivos, por tanto, pueden ser una fuente de ventaja competitiva (AIvarez y Busenitz, 2001).

Las instituciones de apoyo pueden ser para las PyME las facilitadoras del desarrollo o acceso a los recursos que carecen para así superar sus limitaciones $u$ obstáculos. Estas organizaciones también han contribuido a que el empresario se percate de los retos que enfrentan asesorándoles 0 ayudándoles a prepararse para afrontarlos (Lall, 2000). El impacto que tienen las instituciones de apoyo en las PyME pudiera manifestarse en beneficios primarios y secundarios (Wood, 1994). Los beneficios primarios son aquellas mejoras observables en los resultados obtenidos por la empresa y que son mesurables (e.g. incremento en las utilidades o participación en el mercado) (Chrisman y McMullan, 2002). Los beneficios secundarios son producto de un proceso de largo plazo como son las innovaciones con potencial comercial (Burgelman y Sayles, 1986).

\section{Método de investigación}

Este artículo presenta los resultados de un estudio comparativo que utilizó un enfoque histórico para estudiar el desarrollo de ventaja competitiva en algunas PyME que fueron creadas y/o sobrevivieron el periodo de reformas económicas (1979-1999). Para ello, se definió una dimensión comparativa que representó el entorno de las PyME: en el caso de México fue la liberalización y en el Reino Unido, la desregulación. Estos contextos económicos fueron el medio para identificar su impacto y cambios en las PyME para desarrollar ventaja competitiva.

La comparación entre PyMEs se enfocó en el estudio de su capacidad de adaptación, supervivencia y desarrollo de ventaja competitiva, a través de sus estrategias adoptadas en el periodo de estudio. El manejo inicial de la ventaja competitiva en términos de la supervivencia de las PyME ayudó a superar los problemas relacionados con diferentes culturas, niveles de desarrollo económico, su moneda, etc. Así, la supervivencia se convirtió en un concepto con validez que permitió hacer las comparaciones al mismo tiempo que aportó una equivalencia conceptual (Osborne, 1996). La aplicación de este enfoque de la ventaja competitiva ilustra cómo este concepto puede manifestarse de una manera no tradicional. Los aspectos que pueden contribuir al desarrollo de ventaja competitiva fueron explorados cualitativamente con la finalidad de identificar por qué y cómo éstos se convirtieron en factores competitivos para las PyME. En este proyecto el interés primordial se centró en rescatar la experiencia de desarrollo de ventaja competitiva en las PyME tal cual fue experimentada por ellas. Para ello, se desarrollaron y analizaron inductivamente diez estudios de caso que representan a cinco PyME británicas y cinco mexicanas.

Los casos de las PyME mexicanas representan a tres medianas empresas y dos pequeñas empresas. Al menos una empresa representa a cada uno de los principales clusters de calzado: León, Guadalajara y Ciudad de México-Estado de México. Los casos de las PyME británicas representan a cuatro pequeñas em- 
presas y una mediana empresa. Éstas se ubican en diferentes regiones incluyendo la principal región productora de Northamptonshire además de Lincolnshire, Escocia y Essex. El Cuadro 1 presenta una sinopsis de las diez PyME. No se incluyen los casos de las instituciones de apoyo debido a que el objeto de estudio es la PyME. Las empresas seleccionadas para los estudios de caso cubrieron los siguientes criterios:

- El tamaño de las empresas correspondió a la clasificación existente en su país de origen. Según la Secretaría de Economía, en México, una empresa pequeña en el sector industrial tiene entre 11 y 50 empleados y una empresa mediana entre 51 y $250 \mathrm{em}$ pleados (Diario Oficial, 2002). En el Reino Unido, el Departamento de Industria y Comercio establece que una empresa pequeña tiene hasta $49 \mathrm{em}$ pleados y una empresa mediana entre 50 y 249 empleados (DTI, 2001).

- Las PyME experimentaron alguno de los periodos cubiertos por este estudio. El periodo de tiempo para el caso de México consideró como antecedente el periodo de substitución de importaciones (años 70 y parte de los 80) y la liberalización de la economía (a partir de 1986) hasta 1999. Para el caso del Reino Unido fue a partir de la desregulación de la economía en 1979 hasta 1999.

- Se buscó que las empresas fueran exportadoras porque la exportación es considerada un indicador de desempeño competitivo (Buckley et al., 1988). Esta idea sugiere que las empresas han logrado superar o neutra- lizar la fuerte competencia que existe en los mercados internacionales. Este criterio fue deseable mas no restrictivo.

La recopilación de datos se hizo mediante entrevistas personales tanto a los empresarios como a representantes de las organizaciones de apoyo, con el propósito de:

- Identificar los cambios en el ambiente y cómo afectaron a las empresas;

- Comprender cómo el empresario enfrentó las demandas del entorno;

- Identificar las interacciones con las organizaciones de apoyo y los recursos utilizados en el diseño de estrategias que crearon ventajas competitivas; e

- Identificar cómo se conservó la efectividad de las estrategias.

Estos datos fueron complementados con la consulta de documentos, registros históricos y la observación directa. En todos los casos, se aplicaron entrevistas semi-estructuradas que fueron conducidas siguiendo una guía de entrevista para empresarios y para representantes de instituciones de apoyo. Las guías fueron divididas en varios temas relacionados con el entorno, la industria, el empresario, la experiencia del empresario con las organizaciones de apoyo, los recursos de la empresa, la internacionalización, varios aspectos de la cadena y la producción, capacitación, aspectos organizacionales y relacionados con la mano de obra, el papel de las organizaciones de apoyo, fuentes de ventaja competitiva y los obstáculos al crecimiento de la industria en el periodo cubierto por esta investigación. 


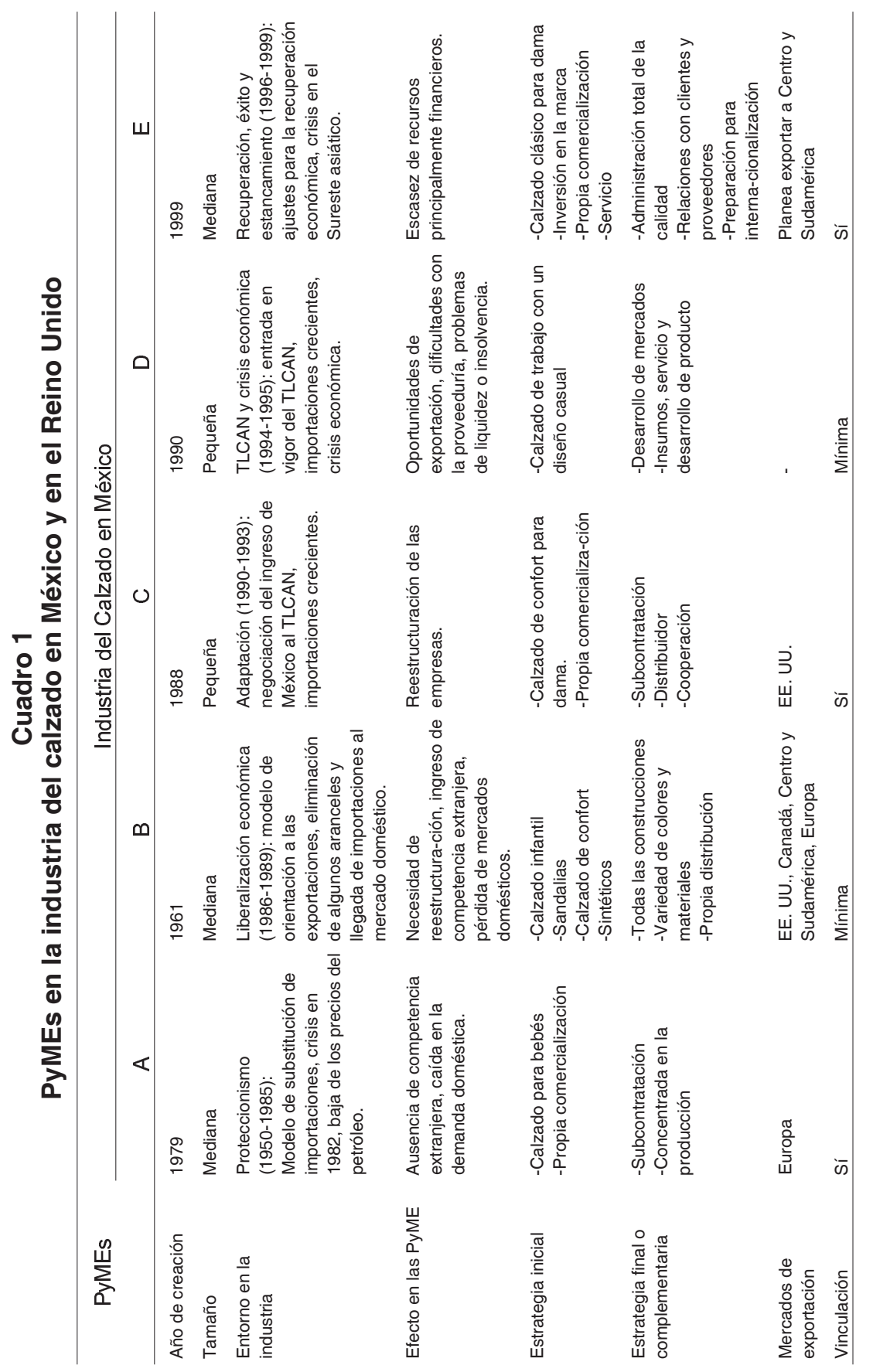




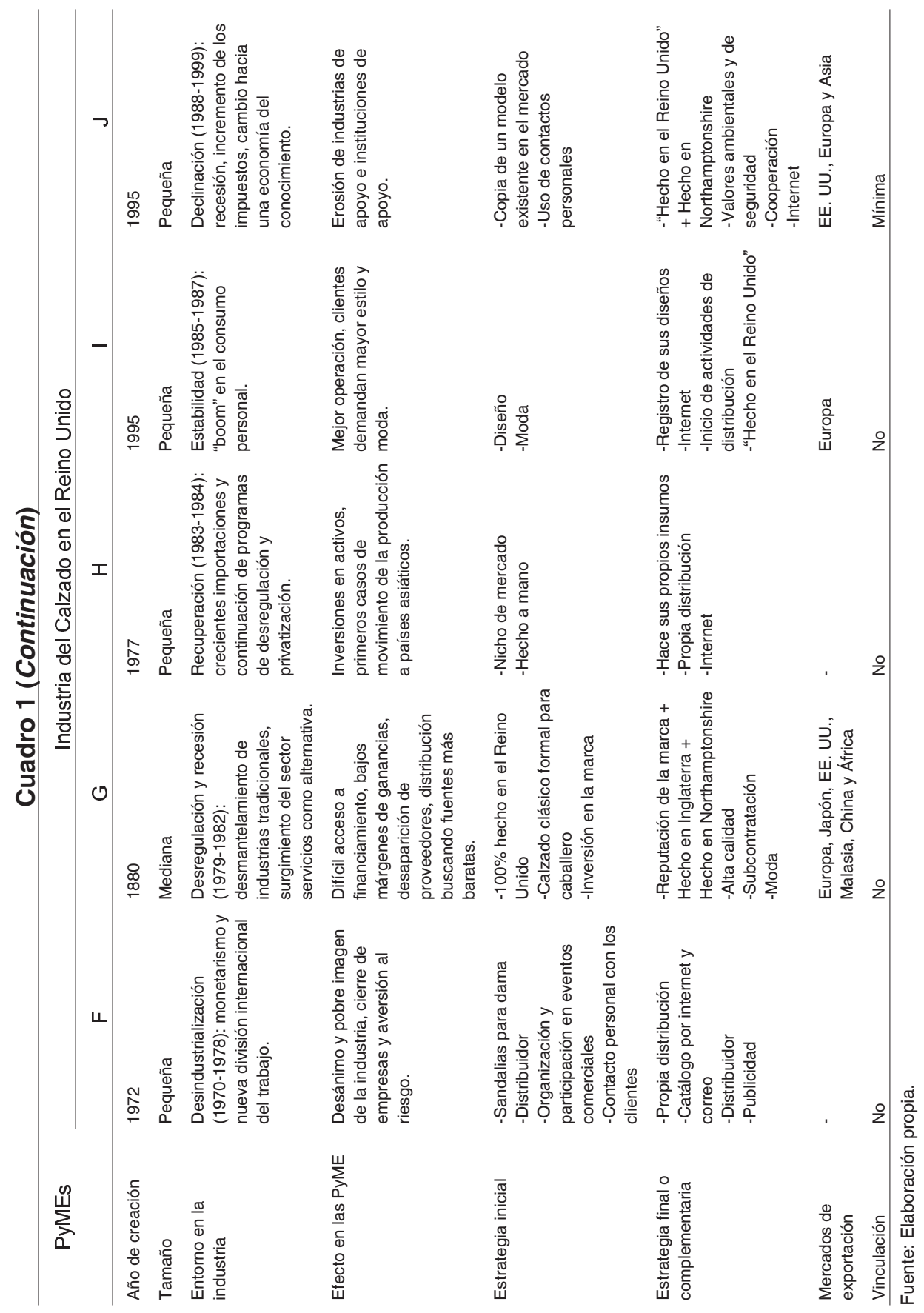


La guía de entrevista se basó fundamentalmente en preguntas abiertas de tipo descriptivo, de contraste y evaluativo, que exploraran las perspectivas, experiencias, conceptos y cambios en la operación de las empresas. Las observaciones en ambos países incluyeron la exploración de los clusters, las instalaciones de las PyME y de las organizaciones de apoyo, con previa autorización.

La consulta de fuentes secundarias incluyó información impresa, reportes y estudios de la industria, revistas especializadas, reportes y estadísticas elaboradas por las organizaciones gremiales, diversas obras publicadas, información pública sobre la industria y hojas electrónicas. La consulta de fuentes secundarias para el caso del Reino Unido también incluyó la colección de documentos sobre la industria en el Museo y Galería de Arte de Northampton además de las colecciones disponibles en la Biblioteca Británica.

El análisis de los datos para cada estudio de caso fue de tipo inductivo y consistió en la clasificación de los datos, examinando los cambios en las empresas y la forma en que éstas se adaptaron mediante la búsqueda de conexiones que identificaran sus alternativas o secuencias de acciones. Los vínculos entre los factores a estudiar guiaron el análisis del cual surgieron definiciones o descripciones de conceptos y procesos relacionados con las características esenciales de la ventaja competitiva en las PyME. A partir de los estudios de caso se construyó el caso de la industria del calzado en cada país. Para tener consistencia y confiabilidad, se desarrollaron una serie de pasos que sirviera de guía en la elaboración del estudio comparativo.
Éste consideró los requisitos y las sugerencias de la literatura existente sobre la elaboración de estudios comparativos. El estudio comparativo de las PyME en ambos países utilizó la misma base de análisis empleada en los estudios de caso. El análisis comparativo se centró en tres niveles: la identificación de las condiciones en las que ocurrieron variaciones en el comportamiento en un periodo específico de tiempo; cómo el comportamiento de las PyME fue regulado por la operación de ciertas fuerzas ambientales; y cómo es que esos cambios ocurrieron.

\section{Resultados}

El análisis de los estudios de casos permitió cumplir con el objetivo del proyecto al identificar cómo las PyME lograron no sólo sobrevivir sino desarrollar ventajas competitivas que les permitieron participar exitosamente en sus respectivos mercados. Debido a limitaciones de espacio, sólo se presentan los dos casos más representativos de cada país, referidos como el caso británico y el caso mexicano. Estos dos casos representan a PyMEs en las que se apreció claramente cómo la ventaja competitiva surgió mediante la adaptación al entorno y a las interacciones entre el empresario, las instituciones de apoyo y los recursos de la empresa inmersos en las estrategias. En ambos casos se introducen citas textuales de los empresarios obtenidas de las entrevistas. Inicialmente cada caso será presentado de forma individual, para posteriormente, indicar cuáles fueron las similitudes y diferencias que se encontraron. 


\section{Caso británico}

Este caso representa a una empresa pequeña que fue creada en 1972 con la finalidad de atender el segmento medio-alto de sandalias en piel para dama hechas a mano. Antes de iniciar su propio negocio, el empresario desempeñó el cargo de gerente de entrenamiento en una compañía grande productora de calzado. Esta experiencia y su deseo por independizarse lo llevaron a crear su propio negocio, mismo que se convirtió en una forma de vida. Hasta hoy, esta actividad le brinda el placer de hacer zapatos y conservarse independiente. Esta libertad se ha extendido al grado de administrar su empresa bajo su estilo muy personal, influido por el movimiento hippie de los 70 . Esto le ha dado autenticidad a su empresa. Cuando la empresa inició sus operaciones, la industria del calzado en el Reino Unido empezó a enfrentar severas dificultades:

'en este país la ausencia total de inversión [y] la pobre administración [hizo] que los negocios familiares pequeños sobrellevaran [su negocio durante] los años 60 y 70 para luego decaer completamente... Me parece que esto se debe en parte a que fuimos unos de los primeros países en industrializarse y teníamos que ser uno de los primeros en desindustrializarse' (Smith, entrevista 2004).

El posicionamiento de la empresa en la industria del calzado fue gracias al uso de técnicas manuales en la elaboración de calzado "personalizado" y el desarrollo de su propia marca. Cada par de sandalias era hecho de manera que se ajustara a las características de los pies del cliente. Este modo de producción permitió a la empresa manejar bajos niveles de inventario y desarrollar la flexibilidad necesaria. De esta forma, la empresa sólo requería tecnología simple pues el insumo principal era la destreza manual para la fabricación del calzado. Las características de los productos también fueron el principal medio para competir con otros fabricantes. La variedad de colores fue un factor primordial para la diferenciación de sus productos. En los 70 la variedad de colores en calzado era altamente demandada pero muy pocos productores podian satisfacer esta necesidad. Otra estrategia que el empresario aplicó fue el ofrecimiento de maquilar calzado a otras compañías con la finalidad de ampliar sus oportunidades de negocios. Sin embargo, esta oportunidad rara vez ocurrió porque, según el empresario, la colaboración entre los fabricantes de calzado tenía que ver más con 'un comportamiento de caballeros con los competidores' (Smith, entrevista 2004). Además, el dueño ofrecía a sus clientes la posibilidad de reparar el calzado adquirido en su negocio, esto con la finalidad de ofrecer un servicio que generara valor agregado. Esta idea surgió de la observación del dueño con respecto al apego que llegan a desarrollar las personas a ciertos objetos personales resultándoles difícil desecharlos cuando éstos han cumplido con su vida útil.

La importancia que el dueño asignó a las personas, tanto a sus clientes como a sus empleados también se convirtió en un factor estratégico. Por un lado, los clientes apreciaban el contacto cercano y personalizado con la empresa, lo que contribuyó a que desarrollaran una leal- 
tad muy sólida. Esto se tradujo en un factor que ayudó a la empresa a sobrevivir en los periodos más difíciles por los que atravesó la industria en los 80 y 90 . La compañía controló su propia distribución mediante la venta postal por catálogo que fue todo un éxito en los 70 y 80 cuando la industria tenía un fuerte problema con la distribución. El empresario explicó que los distribuidores buscaban fuentes de proveeduría cada vez más baratas que ayudaran a mantener bajos niveles de inventario y así poder generar elevados márgenes de ganancia. La estrategia de venta postal por catálogo permitió tener contacto directo con los clientes y ganarse su confianza. La supervivencia de la empresa ante un entorno tan cambiante fue explicada por el dueño como una cuestión de

'exposición, personalidad y espíritu. Sentimos que, como sobrevivientes, crecimos demasiado y aún estamos aquí. El hecho de que seamos un negocio tan pequeño y personal [hace que] sepa todos los nombres de mis clientes....Muchos de ellos han estado ahí por 30 años..., es por este tipo de autenticidad que siguen llamándonos... aún el perro ladrando en el fondo es parte de la experiencia [de los clientes con mi empresa]. Si no tuviéramos ese contacto con los clientes, habríamos desaparecido a principios de los 90' (Smith, entrevista 2004).

El fortalecimiento de la marca ayudaría a sus clientes a identificarse con ella y con los productos de la empresa. La marca era importante porque 'es la que dicta el mercado... los clientes compran la marca.... como algo que tiene un valor para ellos' (Smith, entrevista 2004). La fa- bricación de calzado es estacional y el empresario reconocía que su producto era uno de los más afectados por esta condición. Con el fin de mantener un nivel de ventas en la temporada otoño-invierno, desarrolló como estrategia complementaria la distribución de calzado invernal de buena calidad a precios muy competitivos en la década de los 80 . Adicionalmente, las actividades de desarrollo del producto continuaron para incrementar el número de estilos, mismos que eran exhibidos en eventos comerciales y en las propias pasarelas que el empresario organizaba.

En la experiencia del empresario, la industria del calzado empezó a dar señales de declinación en los 90. Las industrias productoras de insumos fueron desapareciendo gradualmente. Los pocos proveedores que sobrevivieron perdieron el interés de hacer negocios con las empresas de menor tamaño. Éstas carecían de suficiente poder negociador para conseguir buenos términos con los proveedores. Aun cuando el dueño tenía la disposición de pagar un precio más alto a cambio de insumos de buena calidad, los proveedores se rehusaron a vender pequeñas cantidades. De esta forma, los planes de expansión del empresario se cancelaron porque, para poder concretarlos, necesitaba del apoyo de sus proveedores. Así, en la opinión del empresario 'la industria del calzado se contrajo... debido a la reducción de proveedores de componentes, toda la infraestructura de proveeduría para pequeños productores había desaparecido' (Smith, entrevista 2004). También ocurrió la desaparición gradual de industrias de apoyo, tales como la producción de revistas especializadas en aspectos de calzado, mismas 
que a pesar de que eran una valiosa fuente de información, su publicación cesó. Este escenario se complicó por los costos elevados de la mano de obra en la industria y por la pérdida de know how con el retiro de los empleados.

Esta situación se agravó con el escaso reemplazo de la fuerza de trabajo por nuevas generaciones quienes veían poco atractiva a la industria del calzado. A este respecto, el empresario tuvo el acierto de desarrollar una buena relación de largo plazo con sus trabajadores mediante el contacto personal con ellos y el ofrecimiento de contratos anuales de trabajo. Este tipo de contratos eran raros en la industria puesto que se había vuelto práctica común el ofrecimiento de contratos laborales de entre tres y seis meses. De esta forma, los trabajadores también desarrollaron lealtad a la empresa.

En la década de los 90 la empresa modificó su estrategia de distribución de calzado para la temporada invernal al sustituir el producto anterior por un calzado de calidad mayor y que gozaba de la buena reputación de una marca europea. Esta marca dio mucha mayor confianza a sus clientes quienes valoraron su contenido en diseño. Sin embargo, aún en la temporada baja de venta de sandalias, el empresario estimulaba la demanda mediante el ofrecimiento de descuentos si los clientes ordenaban su producto durante el otoño o el invierno. El empresario redujo su inversión en desarrollo de producto cuando la industria sufrió una contracción considerable. Esta actividad se substituyó por la realización de modificaciones menores en sus modelos existentes para mantenerlos acordes a las tendencias de la moda. Por cuestiones de costo y tiempo disponible, el empresario dejó de participar en eventos comerciales y canceló la organización de pasarelas.

El incremento en su base de clientes se dio gracias a la recomendación personal que hacian sus clientes en su círculo de amistades. El internet se adoptó como un medio para crear una plataforma en la que la empresa anunciara su catálogo y ampliara su clientela. La inversión en el fortalecimiento de la marca continuó mediante su publicidad en revistas y otros medios impresos. La empresa siguió controlando su propia distribución mediante la venta postal por catálogo y la apertura de dos pequeñas tiendas para la venta de sus productos.

A pesar de ser una empresa pequeña, su negocio creció considerablemente, el dueño comprendió que el tamaño de la empresa había facilitado su adaptación a los cambios ocurridos en la industria. El empresario manifestó que el desempeño financiero del negocio siempre fue satisfactorio y hasta rebasó sus expectativas si se considera que él no tenía como objetivo principal la generación de utilidades. El dueño comentó que la independencia que siempre buscó a través de su empresa le permitió:

'no obsesionarse con obtener utilidades [financieras] para poder sobrevivir; no tengo a quien rendirle cuentas anualmente... somos un tipo de negocio inusual, sólo con tomar en cuenta nuestras raíces, éstas establecen nuestras políticas. Posiblemente por adoptar un enfoque más relacionado con un estilo de vida generamos un negocio cómodo que nunca tuvo pérdidas, sólo producimos lo suficiente para continuar' (Smith, entrevista 2004). 
El empresario señaló que el principal cambio ocurrido en las organizaciones de apoyo fue que empezaron a apoyar a los distribuidores de calzado, en lugar de continuar apoyando a los productores. Él conocía a varias de estas organizaciones, sin embargo, decidió mantenerse distanciado de ellas para preservar su independencia:

'Pienso que había mucho apoyo disponible hace mucho tiempo. En los 70 aún había ayuda para las pequeñas empresas e industrias, tanto para iniciar como para continuar con sus operaciones... mucho de ese apoyo desapareció en los años de Margaret Thatcher [19791990]' (Smith, entrevista 2004).

Una vez que se ha expuesto el caso británico, proseguiremos con la presentación del caso mexicano.

\section{Caso mexicano}

Este caso representa a una empresa mediana de tipo familiar dedicada a la producción de calzado en piel. La compañía fue fundada en 1979 tratando de explotar la experiencia y el conocimiento previo de la familia en esta industria. Esta organización vivió los últimos años del régimen de substitución de importaciones y el periodo de reforma económica. En sus orígenes, sus principales objetivos eran hacer un negocio redituable mediante la fabricación de calzado para bebés bajo una marca propia y ofrecer buenas condiciones de trabajo a los trabajadores. El propietario indicó que en los primeros años de operación, la empresa tuvo un desempeño regular llevándola al paulatino desarrollo de mercados en otras regiones del país. Se invertía en el desarrollo del producto y éste se comercializaba en boutiques para bebés. Según el empresario, sus actitudes y comportamiento pasivo influyeron en cierto grado en la operación de la empresa:

"el "ritmo/paso" de mi compañía era muy lento y yo sólo hacía lo que podía según mi poco y pobre conocimiento o entendimiento del mundo. Yo empezaba en la industria y no acostumbraba viajar, ni conocer las tendencias de la moda o los cambios en el gusto del cliente, [tampoco] conocer nuevas tecnologías o cómo producir mejor. Pero poco a poco el mercado me ahorcó y me presionó a cambiar. Entonces decidí cambiar.' (Labastida, entrevista 2004).

Cuando los primeros competidores extranjeros entraron a los mercados domésticos en 1985, la empresa experimentó la contracción de su mercado y el debilitamiento de la marca. Los primeros intentos por neutralizar el impacto de la competencia extranjera ocurrieron a finales de los 80 y principios de los 90 . La estrategia se enfocó en la reducción de costos mediante la colaboración con otras empresas para hacer compras conjuntas y desarrollar proyectos conjuntos de comercialización.

Estos esfuerzos sirvieron para aliviar las dificultades pero fueron temporales debido al frágil compromiso de algunos participantes en estos esquemas de colaboración. En la opinión del empresario 'los primeros esfuerzos de colaboración no fueron exitosos porque cada [empresario] velaba por sus propios intereses' (Labastida, entrevista 2004). El dueño encontraba cada vez más difícil manejar todos los aspectos del negocio, particularmente la comercialización y la recu- 
peración de las cuentas por cobrar. Asimismo, la empresa resentía con mayor intensidad las presiones del entorno de la industria. El empresario señaló que a principios de 1994:

'mi empresa tenía todo, estaba abierta al mercado, tenía mi propia marca, hacía mis propios desarrollos de producto y eso era suficiente para mí. Pero de pronto, el mercado se contrajo [nuevamente] y sólo las marcas fuertes sobrevivieron mientras que las débiles, como la mía, morían lentamente' (Labastida, entrevista 2004).

El debilitamiento de la empresa y la "muerte" de la marca amenazaron gravemente su futuro. El empresario explicó que sus esfuerzos no eran suficientes para la magnitud de la situación en la que se encontraba. Le tomó un largo tiempo comprender que la supervivencia de su empresa requería una reestructuración más profunda. La situación empeoró con la crisis financiera en diciembre de 1994 cuando muchas empresas quedaron en banca rota y la recuperación de la cartera vencida fue imposible.

En consecuencia, el dueño tuvo que suspender temporalmente la operación del negocio y observar profundamente hacia el interior de su empresa. De este análisis, el empresario se dio cuenta que la existencia de su empresa y la forma adecuada de lidiar con la competencia dependía de 'mirar cuidadosamente al interior de [su] empresa y estar al tanto de la producción, eficiencia, de la competitividad de los productos ofrecidos en términos de precio, calidad, entrega, diseño y el trabajo en equipo' (Labastida, entrevista 2004). Era necesario identificar cuáles eran las alternativas que le quedaban a su empresa para sobrevivir en la industria. El dueño decidió prescindir de aqueIlas actividades que no eran una fortaleza de la empresa y concentrarse en su actividad medular: la manufactura.

El empresario consideró que la colaboración era la única alternativa viable para superar las dificultades y continuar aprendiendo cómo operar su negocio de una mejor manera: 'Es necesario un gran cambio de mentalidad. Pienso que para que esto ocurra, desafortunadamente tenemos que experimentar severas dificultades. Entonces, esto nos obliga a aprender de los shocks que sufrimos y tratar de encontrar las alternativas para solucionar nuestros problemas' (Labastida, entrevista 2004).

El empresario se acercó a una de las empresas más grandes de la industria y establece un acuerdo de subcontratación en el que la empresa produciría, inicialmente, pequeños volúmenes de cualquier tipo de calzado en piel para la empresa grande. Esta medida eliminó los problemas que la empresa tenía con la proveeduría al ganar acceso a los proveedores de la empresa grande, al igual que los problemas de comercialización pues la empresa grande se encargaría de esta actividad.

Una de las ventajas de esta estrategia fue que 'mediante el acercamiento a una de las firmas líderes en la industria, hemos aprendido de su experiencia y ésta es la manera en que nosotros también podemos crecer, y con el tiempo, nosotros también podremos ayudar a otras empresas' (Labastida, entrevista 2004). El apoyo ofrecido por la empresa grande produjo notables mejoras en las actividades de producción de la empresa en 
cuestión. Éstas últimas desarrollaron flexibilidad suficiente para adaptarse a los cambios necesarios cuando fueran requeridos. Las mejoras también derivaron de los programas de entrenamiento y de las asesorías recibidas de la Cámara local de calzado. Estos esfuerzos fructificaron y contribuyeron en la eficiencia, reducción de desperdicios, mejoras en calidad y entrega, mejor planeación, en el establecimiento de programas y nuevos esquemas de trabajo, la implantación de sistemas computarizados, mejor controly generación de información valiosa para la toma de decisiones.

El empresario reconoció un cambio en su mentalidad ya que empezó a participar en visitas comerciales a otros países. Estas experiencias abrieron más su mente al aprender de ellas y crear conciencia sobre la importancia de las tendencias de la moda. Los productos de la empresa empezaron a ser exportados a través de la empresa grande a finales de los 90. La internacionalización indirecta contribuyó al desarrollo de la capacidad de competir en los segmentos de mercado medio-alto. Además, la compañía también ganó otros contratos de producción para una marca famosa en Europa. Así, la empresa inició sus actividades de exportación a mercados europeos.

Los cambios también se vieron reflejados en la administración de los recursos humanos; el empresario adoptó un contacto más cercano con su fuerza de trabajo y la capacitación se convirtió en una actividad continua. Todos los empleados incluyendo al dueño empezaron a recibir entrenamiento y capacitación en aspectos operativos, la filosofía de calidad total y trabajo en equipo. Las activi- dades de reclutamiento de personal enfatizaron el reclutamiento de personas abiertas al aprendizaje y a la cooperación.

El empresario se dio cuenta que la capacidad de adaptación de la empresa dependía de él mismo, se convenció que él tenía que poner el ejemplo para poder inspirar a sus empleados. Su comportamiento fue entonces más activo y determinado en trabajar no sólo por la supervivencia de su empresa sino también por la de la industria. Esto lo llevó a convertirse en un miembro activo de la Cámara de la Industria del Calzado de Guanajuato. De esta forma, el papel del empresario se transformó en la fuerza motriz y en el "educador" de su fuerza de trabajo. Su compromiso fue enseñar a sus empleados nuevas formas de hacer su trabajo, crear conciencia de la importancia de la disciplina y el orden para hacer las cosas, así como el valor del trabajo en equipo. En esta forma, el dueño siente que asume su compromiso social para mejorar su comunidad local:

'La disciplina y los valores que les enseñemos a nuestros trabajadores pueden llegar hasta sus hogares y es posible que nuestros trabajadores, a su vez, impacten en sus colonias y así se pueden mejorar sus comunidades... '(Labastida, entrevista 2004).

De los casos expuestos se aprecia que el entorno ejerció una gran influencia en las relaciones entre el empresario, las organizaciones de apoyo y el potencial competitivo de los recursos. Los cambios del entorno pusieron a prueba la visión del empresario y la manera en que administraba su empresa, a su vez, la respuesta del empresario a las exigencias 
del ambiente dependieron de su percepción y habilidad para adaptarse. Al observar el comportamiento de estos factores por el periodo 1979-1999 se observó que los cambios ocurridos en el ambiente de las PyME modificaron los siguientes aspectos:

- La forma en que utilizaron sus recursos;

- La apreciación y uso de los programas de apoyo;

- El comportamiento del empresario (principalmente en el caso de México) $y$

- La forma en que identificaron las oportunidades e interpretaron las amenazas.

Esto hizo que los empresarios prestaran mayory más directa atención al potencial interno de sus empresas con el fin de identificar sus capacidades medulares y explotarlas estratégicamente. EI potencial competitivo de los recursos se modificó porque inicialmente las estrategias se basaban en aspectos cuyo potencial se erosionaba con el tiempo (e.g. tipo de material, variedad de modelos). Éstos se convirtieron en un requisito para "asegurar" su supervivencia.

La capacidad de competir se potenció cuando las estrategias se basaron principalmente en los intangibles como diferenciadores de la empresa. Éstos fueron usados para complementar las estrategias básicas y así las PyME pudieron competir mejor y/o neutralizar a sus competidores. Para lograr un potencial competitivo fue necesario invertir en el desarrollo de nuevos recursos o en incrementar el potencial de los existentes (e.g. diseño, desarrollo de relaciones con pro- veedores, con otros productores y/o distribuidores). Por tanto, estos recursos representan medios endógenos para lograr ventaja competitiva. Además, los resultados apoyan la propuesta de Man et al. (1998) quienes sugieren que la adaptación es un medio de mantenerse competitivo.

En algunos casos, el acceso a nuevos recursos, el mejoramiento de los existentes o el desarrollo de nuevas capacidades fueron facilitados por las instituciones de apoyo. En algunos casos, la interacción entre las PyME y estas instituciones ocurrió de manera intermitente. La relación entre ambas no fue muy cercana debido, en muchos casos, a la desconfianza de los empresarios en este tipo de organizaciones. Esto ocasionó el establecimiento de una relación frágil y a la creación de programas que no necesariamente respondian a las necesidades de las PyME. Por esta razón, el vínculo careció de un ciclo continuo de retroalimentación que contribuyera a su fortalecimiento.

La contribución del dueño a la ventaja competitiva de la empresa inició con el mejoramiento del análisis del ambiente que lo hizo más consciente de las condiciones del entorno. Este grado de atención no sólo se relacionó con la identificación de las oportunidades o amenazas, en este proyecto también incluyó preocupaciones éticas y sociales que fueron incorporadas en las estrategias. Esto a su vez, llevó al empresario a desarrollar un conocimiento más profundo de su empresa. Así, el empresario pudo identificar dónde radicaba su potencial competitivo, cuáles eran sus recursos valiosos y cómo combinarlos junto con sus valores en las estrategias. Esta situación involucró un 
proceso de aprendizaje tanto de las experiencias propias como de terceros, creatividad, atención, cooperación, intuición, adopción de nuevos retos, la mejora en la operación y adaptación de la empresa. Los valores del empresario, su iniciativa y comportamiento proactivo establecieron un marco de referencia para la operación de la firma convirtiéndose en activos valiosos para potenciar las estrategias.

La contribución de las organizaciones de apoyo a la ventaja competitiva de las PyME puede ocurrir indirectamente y sólo hasta cierto punto. Sin embargo, esto no obscurece su papel como facilitadoras de recursos. El apoyo institucional contribuyó al desarrollo de habilidades del empresario y a hacerlo consciente de las oportunidades y retos. De estos resultados se desprende que los tres factores involucrados en el modelo propuesto de desarrollo de ventaja competitiva en PyMEs contribuyen hasta cierto punto, y cada uno en diferente medida, a la creación de ventaja competitiva.

\section{Similitudes}

Los resultados señalan que a pesar de la existencia de sectores o empresas excluidas de nuevos modelos económicos, éstas intentarán sobrevivir y alcanzar niveles competitivos que las mantengan exitosamente en el mercado. Desde el punto de vista emprendedor, estas condiciones abrieron oportunidades de negocios adecuadas a las PyME. Éstas al explotar sus ventajas relacionadas con su tamaño pueden incluso contribuir a la existencia de la industria.

La observación y aprendizaje de las experiencias que ocurrían a otras em- presas en la industria hizo que las PyME aplicaran estrategias complementarias que reforzaron su estrategia básica de nichos de mercado. Las estrategias complementarias dieron ventajas a las empresas para sobrevivir aún cuando la industria desaparecía paulatinamente. Estas estrategias se centraron en explotar aspectos como la lealtad del cliente, mar$\mathrm{ca}$, relación con los empleados, entre otros. El proceso de aprendizaje fue útil para los empresarios porque les ayudó a identificar cuáles eran sus recursos y actividades clave.

\section{Diferencias}

Es posible que la velocidad a la que los cambios económicos ocurrieron en ambos países influenciara el tipo de relaciones entre los empresarios, las instituciones de apoyo y las estrategias de las empresas. En el caso mexicano, el proceso de liberalización transformó rápidamente el entorno de negocios. Los cambios fueron drásticos e inesperados por lo que los empresarios no estaban preparados para responder adecuadamente y la confusión y el comportamiento reactivo predominaron. Este tipo de reacción fue común en aquellos empresarios que experimentaron todo el proceso de reforma económica y, por tanto, su proceso de adaptación tuvo mayores complicaciones. Este comportamiento cambió cuando los empresarios fueron más proactivos en:

- La creación de oportunidades,

- El fortalecimiento de sus recursos,

- El desarrollo de nuevas capacidades $y$ habilidades, $y$ 
- Abandonar aquellas actividades en las que la empresa no era competitiva.

En contraste, el proceso en el Reino Unido aparentemente ocurrió a un ritmo más lento. Los empresarios británicos desarrollaron tempranamente conciencia de cuáles eran sus recursos valiosos sobre los cuales basaron sus estrategias. Ellos parecian estar mejor preparados debido a la previa identificación de oportunidades en el mercado donde podían competir mejor y sus recursos podían ser utilizados estratégicamente. Una diferencia notable entre las PyME de ambos países es su relación con las instituciones de apoyo. En el caso del Reino Unido, esta relación es casi inexistente, y esto se explica parcialmente por la desaparición de la infraestructura de apoyo a la industria. Sin embargo, a esta escasa vinculación también contribuye el escepticismo del dueño y su comportamiento basado en la independencia. Por su parte, las PyME mexicanas, algunas las consideraron un apoyo valioso, $y$ por esta razón mantenían un contacto directo con ellas. Las PyME que habían recibido ayuda, se beneficiaron del apoyo para la exportación y el mejoramiento de sus operaciones. El apoyo incluyó asesoría, financiamiento y programas de mejoramiento de la calidad y de otros aspectos administrativos que resultaron en una mejor operación de la empresa. En ambos casos, y en congruencia con lo señalado por Alvarez y Busenitz (2001), fue evidente que la adaptación de las empresas estuvo determinada en gran medida por el aprendizaje y acumulación de experiencia.

\section{Conclusiones}

Las PyME tuvieron que encontrar su propia forma de integrarse a la nueva economía y al entorno competitivo global. El proceso de reforma económica en el Reino Unido se desarrolló gradualmente y se caracterizó por la eliminación paulatina de todo apoyo a las industrias de uso intensivo de mano de obra. Esta situación produjo la erosión de los clusters del calzado y amenazó la supervivencia de las PyME en esta industria. En el caso de México, el proceso de liberalización transformó rápidamente el entorno de negocios en el que los productores de calzado operaban con anterioridad. La supervivencia de las PyME dependía de su habilidad para mejorar su funcionamiento y producir bienes que cumplieran estándares internacionales.

La capacidad de adaptación desarrollada por las PyME en ambos países para enfrentar su entorno se convirtió en un recurso valioso que contribuyó a su flexibilidad para responder a las demandas del mercado y el ambiente.

Este estudio permitió observar que los cambios en el modelo económico de un país conducen al diseño de políticas y una provisión selectiva de apoyo que se centran en favorecer particularmente a determinados sectores económicos o empresas. Los resultados también señalan que el reto que enfrentan las empresas, principalmente las menos favorecidas por las políticas económicas, es su supervivencia y el logro de niveles competitivos que las mantengan como competidores exitosos en sus respectivos mercados. Esto fue posible a través de 
los medios que las empresas tuvieron a su alcance $o$ aquellos que eran accesibles mediante esquemas de cooperación y asistencia por parte de diversas organizaciones para desarrollar ventajas competitivas.

Por ello, el empresario, los recursos de la empresa y los obtenidos de las instituciones de apoyo juegan un papel importante en el desarrollo de ventaja competitiva en las PyME. Se pudo apreciar que las nuevas reglas económicas fueron hasta cierto punto asimiladas e incorporadas a las actividades y forma de operar de las PyME. Sin embargo, los empresarios encontraron la forma de acomodar sus intereses y objetivos personales en los objetivos de negocios, conduciendo su empresa de acuerdo a sus valores personales.

La evidencia empírica sugiere que la ventaja competitiva resultó del aprendizaje de las experiencias propias y ajenas. Esta experiencia facilitó la identificación de recursos estratégicos explotados en estrategias adecuadas a las demandas del entorno. Además, los resultados indican que los recursos básicos sirvieron de base para el desarrollo de una base competitiva mínima necesaria para la supervivencia de la empresa. Así, el desarrollo de ventaja competitiva en el corto plazo puede relacionarse con la supervivencia de la empresa bajo entornos muy dinámicos. Pero una vez superada esta etapa se buscó mejorar la posición en el mercado mediante la implementación de estrategias complementarias. En algunos casos, estas medidas resultaron en la internacionalización de las empresas. Los resultados de este estudio han permitido una perspectiva diferente de la ventaja competitiva en las PyME. Éstas incorporaron nuevos factores a sus estrategias tales como el servicio, flexibilidad, aspectos éticos y sociales. Estos intangibles las convirtieron en participantes exitosas en el mercado.

Este resultado representa un área que debe ser explorada más a fondo. Sería interesante examinar si este concepto es transferible a otras industrias y si se relacionan con alternativas estratégicas disponibles para las PyME. Los retos para las PyME continúan siendo la competitividad, la creatividad en la explotación de recursos valiosos en estrategias efectivas, y el desarrollo de nuevos recursos y capacidades que fortalezcan su capacidad de competir. El estudio de las PyME en la industria del calzado representa un caso interesante que podría aportar enseñanzas y experiencias a otras PyME en industrias diferentes.

Por último, cabe señalar que los resultados de este trabajo no pueden ser generalizados porque sólo representan la experiencia de unas cuantas PyME en la industria del calzado que no necesariamente es compartida por otros productores de calzado. Los resultados y sus implicaciones son específicos a la industria; quizás si este estudio se llevara a cabo en otras industrias, las conclusiones serian diferentes.

\section{Referencias Bibliográficas}

Alvarez, Sharon y Busenitz, Lowell (2001). 'The entrepreneurship of ResourceBased Theory,' Journal of Management, Vol. 27, No. 6, pp. 755-775. 
Andrews, R; Didow, Nick; Peacock, James y Clark, Galahad (1998). 'Economics of world shoe production trends,' consultada el 24 de abril del 2004. Disponible en http://www.unc.edu/ andrewsr/ints092/clark.html.

Barney, Jay (1991). 'Firm resources and sustained competitive advantage,' Journal of Management, Vol. 17, No. 1, pp. 99-120.

Barney, Jay (1986). 'Strategic factor markets: Expectations, luck and business strategy,' Management Science, Vol. 32, No. 10 , pp. 1231-1241.

Buckley, Peter; Pass, Christopher y Prescott, Kate (1988). 'Measures of international competitiveness: A critical Survey,' Journal of Marketing Management, Vol. 4, No. 2, pp. 175-200.

Burgelman, Robert y Sayles, Leonard (1986). Inside Corporate Innovation: Strategy, Structure, and Managerial Skills. Free Press: Nueva York.

Chrisman, James y McMullan, Ed (2002). 'Some additional comments on the sources and measurement of the benefits of small Business assistance programs,' Journal of Small Business Management, Vol. 40, No. 1, pp. 43-50.

Collis, David y Montgomery, Cynthia (1995). 'Competing on resources: Strategy in the 1990s,' Harvard Business Review, Vol. 73, pp. 118-128.

De Oliveira, Eduardo y Evaldo, Jaime (2003). 'Use of resource-based view in industrial cluster strategic analysis,' International Journal of Operations and Production Management, Vol. 23, No. 9, pp. 995-1009.

Diario Oficial de la Federación (2002). Segunda Sección, Secretaría de Economía, 30 de diciembre, consultado en febrero del 2004. Disponible en http:// www.gobernacion.gob.mx/dof/2002/ diciembre/dof_30-12-2002.pdf
Departamento de Industria y Comercio (DTI) (2001). 'Small and Medium Enterprise (SME) definitions', Department of Trade and Industry, Reino Unido, consultado en febrero del 2004. Disponible en http://wwwdti.gov.uk/SME4/define. htm.

Herrera, Sandra; Burr, Caroline y Johnsen, Rhona (2002). 'Competitor networks: International competitiveness through collaboration,' International Journal of Entrepreneurial Behaviour and Research, Vol. 8, No. 5, pp. 239-253.

Kickul, Jill y Gundry, Lisa (2002). 'Prospecting for strategy advantage: The proactive entrepreneurial personality and small firm innovation,' Journal of Small Business Management, Vol. 40, No. 2, pp. 85-97.

Knight, Gary (2000). 'Entrepreneurship and marketing strategy: The SME under globalisation,' Journal of International Marketing, Vol. 8, No. 2, pp. 1232.

Lall, Sanjaya (2000). 'Strengthening SMEs for international competitiveness.' Trabajo No. 44 presentado en el Taller What Makes Your Firm Internationally Competitive, Tercer Encuentro del Foro Mediterráneo de Desarrollo, Cairo, del 5 al 8 de marzo.

Man, Thomas; Lau, Theresa y Chan, K. (1998). 'Conceptualisation of SMEs competitiveness: A focus on entrepreneurial competencies,' Thomas Department of Management. The Hong Kong Polytechnic University, consultado en agosto del 2003. Disponible en http://www.sbaer.uca.edu/Research/1998/ICSB/y004.htm.

Mintzberg, Henry; Ahlstrand, Bruce y Lampel, Joseph (1998). Strategy Safari: The Complete Guide Through the Wilds of Strategic Management. FT Prentice Hall: Harlow, Reino Unido. 
Osborne, S. (1996). Selecting a Methodology for Management Research: Issues and Resolution, Trabajo No. 34, agosto, Public Services Management Research Centre, Aston Business School.

Quinn, James Brian (1996). 'Strategies for Change' en The Strategy Process, Henry Mintzberg y James Brian Quinn (Eds.), pp. 3-10. Edición Internacional, tercera edición. Prentice Hall Internacional: Londres.

Ray, Sougata (2003). 'Strategic adaptation of firms during economic liberalisation: Emerging issues and a research agenda,' International Journal of Management, Vol. 20, No. 3, pp. 271-281.

Vokurka, Robert; Zank, Gail y Lund, Carl (2002). 'Improving competitiveness through supply chain management: A cumulative improvement approach,' Competitiveness Review, Vol. 12, No. 1, pp. 14-25.

Whittington, Richard (1993). What Is Strategy - And Does It Matter? Routledge: Londres y Nueva York.

Wood, William (1994). 'Primary benefits, secondary benefits, and the evaluation of small business assistance programs,' Journal of Small Business Management, Vol. 32, No. 3, pp. 6575.

\section{Entrevistas realizadas:}

Labastida, Nicolás (2004). Propietario de la empresa $A$ que representa al caso mexicano; León 14 de julio

Smith, Mark (2004). Propietario de la empresa que representa al caso británico; Lincolnshire 23 de noviembre 2004. 